\title{
CXCL10/CXCR3 overexpression as a biomarker of poor prognosis in patients with stage II colorectal cancer
}

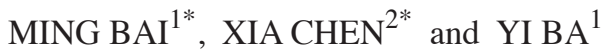 \\ ${ }^{1}$ Department of Gastrointestinal Medical Oncology, Tianjin Medical University Cancer Institute and Hospital, \\ National Clinical Research Center of Cancer, Key Laboratory of Cancer Prevention and Therapy, Tianjin 300060; \\ ${ }^{2}$ Department of Pediatric Hematology Center, Institute of Hematology and Blood Diseases Hospital, Chinese Academy \\ of Medical Sciences and Peking Union Medical College, Tianjin 300041, P.R. China
}

Received February 19, 2015; Accepted July 28, 2015

DOI: $10.3892 / \mathrm{mco} .2015 .665$

\begin{abstract}
The CXCL10/CXCR3 axis of inflammatory mediators is one of the most important groups of chemokine axes, which has been proven to be a lymphocyte-associated metastasis mediator in several tumors. The term inflammatory adhesions refers to tumors found to be attached to the surrouding tissues during surgery, although no cancer cell infiltration is later identified on pathological examination. The aim of the present study was to investigate the clinical characteristics of stage II colorectal cancer (CRC) and determine the correlation between the CXCL10/CXCR3 axis, inflammatory adhesions and prognosis. Clinicohistopathological data were collected from $401 \mathrm{CRC}$ patients who had undergone R0 resection. Statistical analysis was performed with SPSS 17.0 software. Immunohistochemistry (IHC) was applied to measure the expression of CXCL10 and CXCR3 in 71 recurrent CRC patients, 72 non-recurrent CRC patients and 10 samples from normal peritumoral tissues, all retrieved from the Tianjin Medical University Cancer Institute and Hospital, Tianjin, China. Inflammatory adhesions, tumor location and size and the number of high-risk factors for reccurrence were more significantly associated with overall survival (OS) rather than disease-free survival in all the patients as determined by the log-rank and Cox's regression hazard analysis. Further analysis demonstrated that only the presence of inflammatory adhesions $(\mathrm{P}=0.025)$ was associated with the OS of recurrent patients. Patients with recurrence exhibited higher CXCR3 $(\mathrm{P}<0.001)$ and CXCL10 $(\mathrm{P}<0.001)$ expression compared with non-recurrent patients, as determined by IHC. The correla-
\end{abstract}

Correspondence to: Dr Yi Ba, Department of Gastrointestinal Medical Oncology, Tianjin Medical University Cancer Institute and Hospital, National Clinical Research Center of Cancer, Key Laboratory of Cancer Prevention and Therapy, 4th Floor, Building A, Huan Hu Xi Road, Hexi, Tianjin 300060, P.R. China

E-mail:dryiba@gmail.com

${ }^{*}$ Contributed equally

Key words: inflammatory adhesion, CXCL10, CXCR3, colorectal cancer, relapse tion between clinicopathological variables, CXCL10/CXCR3 expression and survival was also analyzed: Inflammatory adhesions and general tumor type (ulcerated vs. elevated) exhibited a significant correlation with CXCR3; however, the expression of CXCL10 was not significantly correlated with tumor location, histological type, size, gender, or preoperative carcinoembryonic antigen and hemoglobin levels. Furthermore, patients exhibiting a high expression of CXCR3 presented with a higher risk of relapse; among those, patients with inflammatory adhesions always exhibited worse survival. However, no such association was identified for CXCL10 expression. In conclusion, CXCR3 expression may be used as a prognostic marker and may contribute to the prediction of clinical outcome in stage II CRC patients.

\section{Introduction}

Colorectal cancer (CRC) is a common malignancy of the gastrointestinal tract, which is fourth in incidence and second in terms of cancer-related mortality in America (1). Several factors and genes are associated with the process of tumor angiogenesis, invasion, growth and metastasis in CRC. A number of stage III/IV patients succumb to metastasis and stage II patients to recurrence, particularly in the liver, lungs and lymph nodes (2). Despite the advances in identifying high-risk factors for recurrence in stage II CRC patients, the benefit from chemotherapy administration remains uncertain (3-5).

Over the last few decades, microsatellite instability (MSI) has been identified as a biomarker in previous clinical trials $(4,5)$, which demonstrated that patients with MSI treated with 5-fluorouracil exhibited a significant survival benefit compared with the non-MSI and the surgery alone groups. Numerous tumor cell-derived factors and microenvironment molecules, such as chemokines, are involved in cancer cell metastasis and migration $(6,7)$. Chemokines (8-10 kDa) are chemotactic cytokines that cause directed migration of numerous cells, including leukocytes, and are induced by inflammatory cytokines, growth factors and pathogenic stimuli. The chemokine-receptor axis allows cells to move towards high local concentrations of chemokines during inflammation, as well as the homeostatic transport of 
lymphocytes and dentritic cells. To date, $>50$ chemokines and 20 seven-transmembrane-domain receptors, which belong to $\mathrm{G}$-protein coupled families, have been identified. One receptor may generally bind to more than one cytokines. A number of human cancers characterized by leukocyte infiltration possess a complex chemokine network that affects tumor cell growth, survival, infiltration, migration and angiogenesis.

Latest research demonstrated that the expression of the majority of chemokines and their receptors, such as CXCL10/CXCR3, CXCL12/CXCR4, CCL21/CCR7 and CCL25/CCR9, is associated with CRC. Dwinell et al (8) reported that $\mathrm{CXCR} 3$ is not present in normal colonic epithelial cells, but in mononuclear cells in the lamina propria. Kawada et al (9) observed that CXCL10 enhances CRC cell survival and gelatinase expression in culture and upregulates cell surface expression of CXCR3. Furthermore, CXCL10 has been found to be overexpressed in several cases of CRC as a Ras target gene (10), although Jiang et al (11) reported opposite findings. It appears that chemokines exert their tumor-associated activies by inducing immune-stimulating and angiostatic effects and constituting the tumor microenviroment. However, the precise role of CXCL10/CXCR3 in solid cancers remains poorly understood.

The aim of the present study was to investigate CXCL10 and CXCR3 expression in stage II CRC, in order to determine its clinicopathological significance and role in disease recurrence and optimise postoperative treatment in patients with stage II CRC.

\section{Patients and methods}

Patients and materials. A series of 401 stage II CRC patients who underwent radical resection at Tianjin Medical University Cancer Institute and Hospital between 2005 and 2009 were included in this study. None of the patients had received preoperative neoadjuvant chemotherapy or radiotherapy. The patients were divided into two groups, the recurrence group (RG) and the non-recurrence group (NRG). We collected paraffin-embedded samples from 71 recurrent cases, 12 non-recurrent cases and 10 normal tissue samples. All the samples were independently reviewed by two pathologists and the histological diagnoses were classified according to the 2010 World Health Organization Classification of Digestive System Tumors (12). The recurrence risk factors of stage II CRC according to the guidelines of the National Comprehensive Cancer Network included poor differentiation, lymph node or blood vessel infiltration, intestinal obstruction, $<12$ lymph nodes retrieved, perineural invasion, partial perforation and positive resection margin. The term inflammatory adhesions refers to tumors found to be attached to the surrouding tissues during surgery, although no cancer cell infiltration is later identified on pathological examination.

Immunohistochemical analysis. Tumor samples were collected from the Tianjin Medical University Cancer Institute and Hospital (Tianjin, China), fixed in formalin, embedded in paraffin and sectioned at $4 \mu \mathrm{m}$. A polyclonal rabbit anti-human CXCL10 antibody (cat. no. (C-20) sc-6226; dilution, 1:120; Santa Cruz Biotechnology, Inc., Santa Cruz, CA, USA) and polyclonal rabbit anti-human CXCR3 anti- body (cat. no. sc-101500; dilution, 1:200) were separately added to the sections following deparaffinization, hydration, antigen repair and endogenous peroxidase blocking. Immunoperoxidase staining was performed with the two-step EnVision $^{\mathrm{TM}}$ method (DakoCytomation, Glostrup, Denmark) according to the manufacturer's instructions and visualized with 3,3'-diaminobenzidine (Sigma, St. Louis, MO, USA). The phosphate-buffered saline buffer was used to prepare negative control samples. Cell membrane and cytoplasmic staining were measured for these antibodies. Two pathologists independently counted the positive cells. The 4-tiered scoring system $(-/+/++/+++)$, which took into account the percentage of positive cells and staining intensity, was used in our evaluation. The expression level of a certain target was determined according to the respective median values of a tumor indicator. Lower than the median was defined as 'low expression' and higher as 'strong expression'.

Follow-up. Follow-up data were collected through telephone communication and from the database of the Medical Records Department of our hospital. The time interval from the operative date to clinical relapse was defined as the disease-free survival (DFS), and to death or last follow-up as overall survival (OS).

Statistical analysis. All the data were analyzed using SPSS 17.0 software (SPSS Inc., Chicago, IL, USA). The t-test and analysis of variance were used for numerical variables and the $\chi^{2}$ test for qualitative variables. For survival analysis, survival curves were generated by the Kaplan-Meier method. The univariate survival analysis was performed using the log-rank test and the multivariate Cox proportional hazards model was used to identify the independent prognostic factors. $\mathrm{P}<0.05$ was considered to indicate statistically significant differences.

\section{Results}

Clinicopathological characteristics. A total of 229 male and 172 female patients, aged 7-87 years (mean age, 60.5 years), were initially included in the study. Of these, $54.86 \%(220 / 401)$ had colon cancer, $45.89 \%$ (181/401) had rectal cancer. Patients with colon cancer exhibited a survival advantage compared with those with rectal cancer $(\mathrm{P}=0.026)$. The general classification was ulcerated type in $288 / 401$, elevated type in $111 / 401$, colloid type in $1 / 401$ and infiltrative type in $1 / 401$. The pathological results of all kinds of carcinomas were adenoma, histologically classified as tubular $(n=198)$, papillary $(n=15)$, mucinous $(n=25)$ and mixed $(n=54)$. Of the 401 cases, 22 were classified as well-differentiated, 335 as moderately differentiated and 44 as poorly differentiated. A maximum diameter of $>10 \mathrm{~cm}$ was observed in $4.99 \%$ (20/401) of the cases, with a significantly different DFS compared with cases with smaller tumors $(\mathrm{P}<0.051)$. In 91 cases, inflammatory adhesions to the surrounding tissues were identified, which statistically significantly affected $\mathrm{OS}(\mathrm{P}=0.024)$, but not $\mathrm{DFS}(\mathrm{P}=0.214)$. A backward Cox's proportional hazards regression analysis of the clinicopathological parameters yielded a hazard ratio of 1.823 (95\% confidence interval: 1.093-3.039; $\mathrm{P}=0.013$ ) for adhesion-positive compared with adhesion-negative patients. Patients with more risk factors exhibited shorter DFS and OS 
Table I. Comparison of clinicopathological characteristics in stage II colorectal cancer patients undergoing potentially curative resection.

A, Clinicopathological characteristics

\begin{tabular}{|c|c|c|c|c|c|c|c|}
\hline \multirow[b]{2}{*}{ Variables } & \multirow[b]{2}{*}{$\begin{array}{c}\text { Total } \\
(n=401)\end{array}$} & \multicolumn{3}{|c|}{ Survival status } & \multicolumn{3}{|c|}{ Recurrence status } \\
\hline & & $\begin{array}{c}\text { Alive } \\
(n=325)\end{array}$ & $\begin{array}{c}\text { Deceased } \\
(\mathrm{n}=76)\end{array}$ & P-value ${ }^{a}$ & No recurrence & Recurrence & P-value ${ }^{a}$ \\
\hline Gender & & & & 0.320 & & & 0.681 \\
\hline Male & 229 & 182 & 47 & & 167 & 62 & \\
\hline Female & 172 & 143 & 29 & & 128 & 44 & \\
\hline Age, years & & & & 0.341 & & & 0.721 \\
\hline$\leq 60$ & 199 & 166 & 33 & & 149 & 50 & \\
\hline$>60$ & 202 & 159 & 43 & & 146 & 56 & \\
\hline Location & & & & $0.026^{\mathrm{b}}$ & & & 0.101 \\
\hline Colon & 220 & 187 & 33 & & 169 & 51 & \\
\hline Rectum & 181 & 138 & 43 & & 125 & 54 & \\
\hline General classification & & & & 0.551 & & & 0.515 \\
\hline Ulcerated & 288 & 229 & 59 & & 206 & 82 & \\
\hline Elevated & 111 & 94 & 17 & & 87 & 24 & \\
\hline Other & 2 & 2 & 0 & & 2 & 0 & \\
\hline Tumor size, $\mathrm{cm}$ & & & & $0.031^{\mathrm{b}}$ & & & 0.051 \\
\hline$\leq 10$ & 381 & 312 & 69 & & 283 & 98 & \\
\hline$>10$ & 20 & 13 & 7 & & 8 & 12 & \\
\hline Stage & & & & 0.179 & & & 0.441 \\
\hline IIA & 71 & 59 & 12 & & 55 & 16 & \\
\hline IIB & 323 & 262 & 61 & & 236 & 87 & \\
\hline IIC & 7 & 4 & 3 & & 4 & 3 & \\
\hline Recurrence risk factors & & & & $0.009^{\mathrm{b}}$ & & & $<0.001^{\mathrm{b}}$ \\
\hline None & 75 & 63 & 12 & & 56 & 19 & \\
\hline 1 & 276 & 229 & 47 & & 208 & 68 & \\
\hline$\geq 2$ & 50 & 33 & 17 & & 31 & 19 & \\
\hline Preoperative CEA & & & & 0.838 & & & 0.205 \\
\hline Abnormal & 28 & 23 & 5 & & 18 & 10 & \\
\hline Normal & 373 & 302 & 71 & & 277 & 96 & \\
\hline Anemia & & & & 0.731 & & & 0.952 \\
\hline No & 300 & 242 & 58 & & 221 & 79 & \\
\hline Yes & 101 & 83 & 18 & & 74 & 27 & \\
\hline Family history & & & & 0.528 & & & 0.295 \\
\hline No & 327 & 263 & 64 & & 235 & 90 & \\
\hline Yes & 74 & 62 & 12 & & 58 & 16 & \\
\hline Inflammatory adhesions & & & & $0.024^{\mathrm{b}}$ & & & 0.214 \\
\hline No & 310 & 258 & 52 & & 232 & 78 & \\
\hline Yes & 91 & 67 & 24 & & 63 & 28 & \\
\hline
\end{tabular}

B, Uni- and multivariate Cox proportional hazards model for overall survival in stage II colorectal cancer patients

\begin{tabular}{lccccrr}
\hline Variables & B & SE & Wald & P-value & Exp (B) & 95\% CI \\
\hline Location & -0.622 & 0.250 & 6.184 & $0.013^{\mathrm{b}}$ & 0.537 & $0.329-0.877$ \\
Tumor size & 0.455 & 0.213 & 4.562 & $0.033^{\mathrm{b}}$ & 1.577 & $1.038-2.395$ \\
Recurrence risk factors & 0.432 & 0.206 & 4.395 & $0.036^{\mathrm{b}}$ & 1.540 & $1.029-2.307$ \\
Inflammatory adhesion & 0.600 & 0.261 & 5.298 & $0.013^{\mathrm{b}}$ & 1.823 & $1.093-3.039$
\end{tabular}

ap-values were obtained with the log-rank and $\chi^{2}$ test in Table IA and Cox regression in Table IB. ${ }^{b} \mathrm{P}<0.05$, statistically significant. B, regression coefficient; SE, standard error; Exp, odds ratio; CI, confidence interval; CEA, carcinoembryonic antigen. 
Table II. Correlation between the clinicopathological characteristics of relapsed patients and overall survival.

\begin{tabular}{lc}
\hline Characteristics & P-value \\
\hline Imflammatory adhesions & $0.025^{\mathrm{b}}$ \\
General classification & 0.314 \\
Recurrence risk factors & 0.194 \\
Tumor size & 0.516 \\
Gender & 0.487 \\
Age & 0.931 \\
N stage & 0.266 \\
Preoperative CEA level & 0.218 \\
Preoperative Hb level & 0.295 \\
Tumor location & 0.309 \\
Family history & 0.859 \\
\hline
\end{tabular}

${ }^{a} \mathrm{P}$-values were obtained with $\mathrm{Cox}$ regression. ${ }^{\mathrm{b}} \mathrm{P}<0.05$, statistically significant. CEA, carcinoembryonic antigen; $\mathrm{Hb}$, hemoglobin.

Table III. Immunostaining for CXCL10 and CXCR3 expression in normal and CRC tissues.

\begin{tabular}{|c|c|c|c|c|c|c|}
\hline \multirow[b]{2}{*}{ Variable } & \multicolumn{3}{|c|}{ CXCL10 } & \multicolumn{3}{|c|}{ CXCR3 } \\
\hline & Negative & Low & Strong & Negative & Low & Strong \\
\hline $\begin{array}{l}\text { CRC } \\
(n=143)\end{array}$ & 51 & 55 & 37 & 60 & 69 & 14 \\
\hline $\begin{array}{l}\text { Normal } \\
\text { tissue } \\
(n=10)\end{array}$ & 4 & 6 & 0 & 10 & 0 & 0 \\
\hline P-value & & 0.145 & & & $0.002^{\mathrm{b}}$ & \\
\hline
\end{tabular}

${ }^{\text {a }} \mathrm{P}$-values were obtained with the $\chi^{2}$ test. ${ }^{\text {b }} \mathrm{P}<0.05$, statistically significant. CRC, colorectal cancer.

$(\mathrm{P}<0.05)$. The multivariate Cox analysis indicated that location, tumor size and the number of high-risk factors were independent variables $(\mathrm{P}=0.013,0.033$ and 0.036$)$, as was the presence of inflammatory adhesions, and were all significant prognostic factors for poor OS (Table IA and B). Colon cancer patients with larger tumors who had $>1$ high-risk factor and inflammatory adhesions were more likely to succumb to the disease.

From the follow-up data, we identified 106 patients with relapse or metastasis, with a median DFS of 21.8 months and a median OS of 39.6 months. The follow-up time ranged from 2 to 36 months. Of the 401 patients, 286 survived without evidence of cancer, 39/401 remained alive with recurrent lesions, 67/401 succumbed to CRC and 9/401 succumbed due to other causes.

Inflammatory adhesions. In the RG, only the presence of inflammatory adhesions was associated with $\mathrm{OS}(\mathrm{P}=0.025)$ (Table II). However, there was no such association with the remaining characteristics. A total of $28(28 / 106)$ of the relapsed
Table IV. Immunostaining for CXCL10 and CXCR3 in colorectal cancer (negative expression samples are absent).

\begin{tabular}{lccr}
\hline & $\begin{array}{c}\text { Recurrence } \\
\text { group } \\
(\mathrm{n}=71)\end{array}$ & $\begin{array}{c}\text { Non-recurrence } \\
\text { group } \\
(\mathrm{n}=72)\end{array}$ & P-value $^{\mathrm{a}}$ \\
\hline VXriable & & & $0.001^{\mathrm{b}}$ \\
Low expression & 19 & 36 & \\
Strong expression & 27 & 10 & \\
CXCR3 & & & \\
Low expression & 21 & 48 & \\
Strong expression & 12 & 2 & \\
\hline
\end{tabular}

${ }^{\text {aP }}$-values were obtained with the $\chi^{2}$ test. ${ }^{\text {b }}<0.05$, statistically significant.

patients exhibited inflammatory adhesions surrounding the tumor and the majority survived for <3 years [67.86 (19/28)]. The median survival time of relapsed patients with adhesions was 26.63 months, while in the NRG it was 61.67 months. It is considered that adhesion had been reported previously to OS. It was concluded that the presence of inflammatory adhesions in a proportion of the patients was not associated with recurrence, but was closely associated with OS; however, for patients who presented with adhesions as well as recurrence, the prognosis was worse.

Immunohistochemistry (IHC) in CRC. Immunostaining with anti-CXCL10 or -CXCR3 was considered to be antibody-specific by using the immunising peptide for each antibody as the target. In order to elucidate the association between clinical characteristics and the CXCL10/CXCR3 axis, we examined $143 \mathrm{CRC}$ specimens and 10 peritumoral tissues at a distance of $\geq 5 \mathrm{~cm}$ from the resection margin (normal intestinal epithelia). CXCL10 and CXCR3 exhibited distinct characteristics in each group (Fig. 1).

As determined by IHC, CXCL10 was poorly expressed in 36 out of 72 cases $(50.0 \%)$ in NRG and 19 out of 71 cases $(26.76 \%)$ in RG, as well as strongly expressed in 10 out of 72 cases $(13.89 \%)$ in the NRG and 27 out of 71 cases $(38.03 \%)$ in the RG. We also found that 48 samples (66.67\%) exhibited low CXCR3 expression in the NRG and 21 samples (29.58\%) in the RG, whereas 12 cases $(16.90 \%)$ exhibited high CXCR3 expression in the RG and 2 (2.78\%) in the NRG. In the normal group, none of the normal tissue samples expressed CXCR3, whereas 6 samples poorly expressed CXCL10. The remaining pathological sections were not stained by the specific antibodies (Tables III and IV). There was a significant difference beween normal tissues and CRC RG or NRG in terms of CXCL10 and CXCR3 expression $(\mathrm{P}<0.05)$.

Effect of clinicopathological characteristics and biomarker expression on survival. The univariate variables were discussed and analyzed at the beginning of this research. We aimed to determine the association between prognosis and the expression of CXCL10 or CXCR3 with the assistance of Kaplan-Meier plots. High expression of CXCR3 was found to 

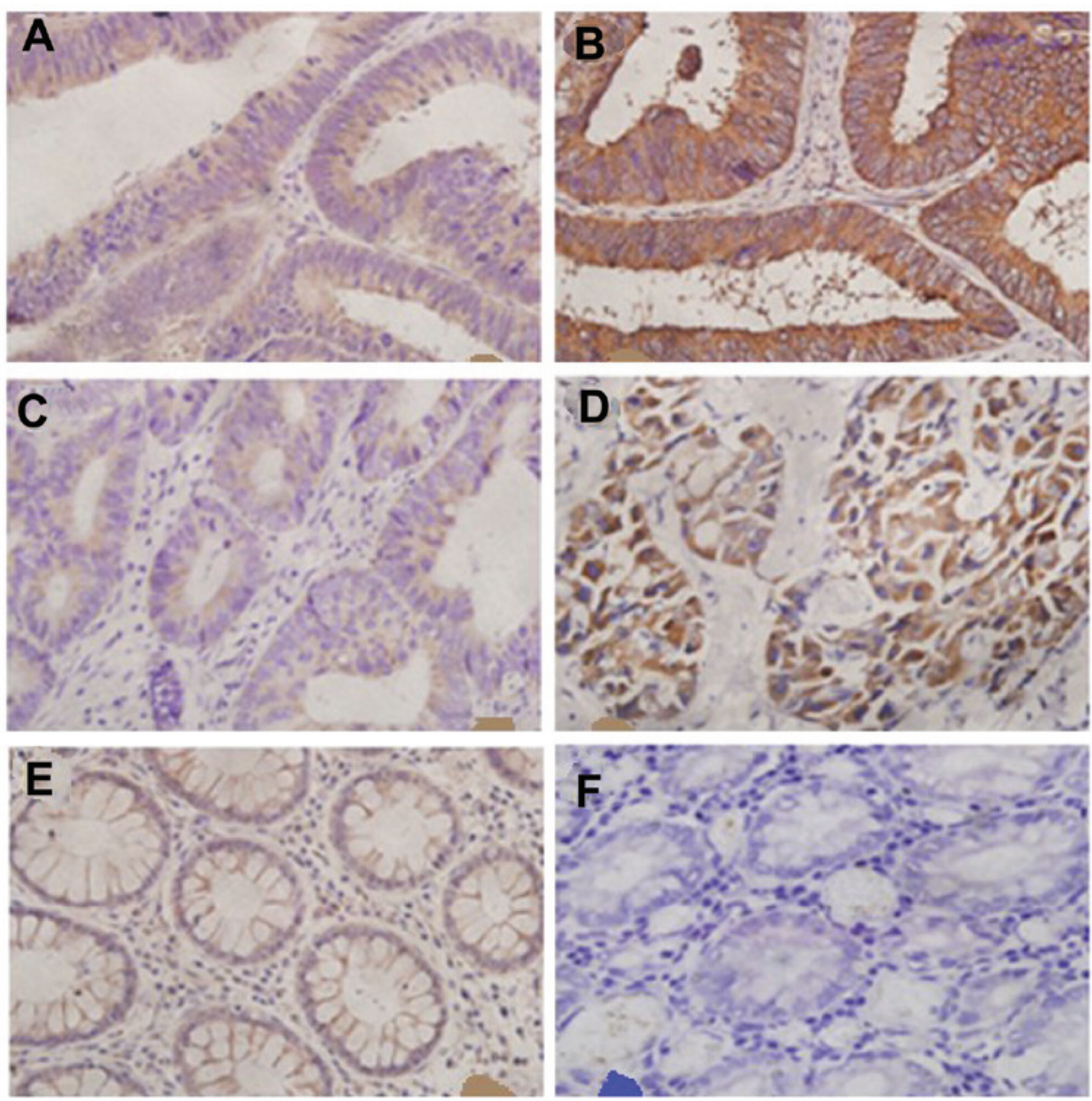

Figure 1. CXCL10 and CXCR3 membranous and cytoplasmic staining in colorectal tumors. (A) Low CXCL10 expression was demonstrated in 19/71 samples in the recurrence group (RG) and 36/72 samples in the non-recurrence group (NRG). (B) Strong CXCL10 expression was observed in 27/71 samples in the RG and 10/72 in the NRG. (C) Low CXCR3 expression was demonstrated in 21/71 samples in the RG and 48/72 in the NRG. (D) Strong CXCR3 expression was demonstrated in 12/71 samples in the RG and 2/72 in the NRG. (E) Low CXCL10 expression was demonstrated in 6/10 normal tissue samples. (F) Negative CXCR3 expression was demonstrated in 10/10 normal tissue samples.

A

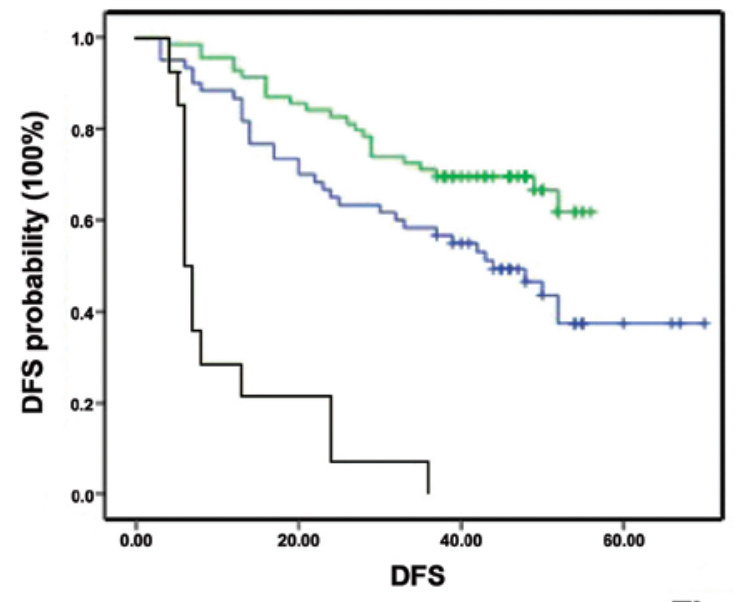

B

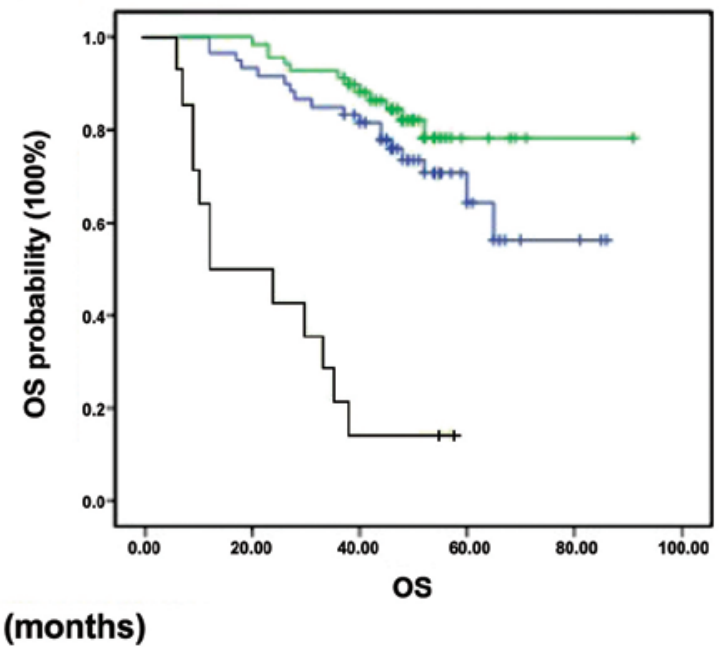

\section{- Negative - High expression - Low expression}

Figure 2. Survival curve of CXCR3 expression. (A) Patients with strong expression ( $\mathrm{n}=14$ ) exhibited significantly shorter disease-free survival (DFS) compared with those with low expression $(n=69, P<0.0001$, log-rank test) and negative expression $(n=60, P<0.0001, \log$-rank test); low vs. negative expression, $\mathrm{P}=0.012$. (B) Patients with strong expression also exhibited significantly shorter overall survival (OS) compared with those with low expression $(\mathrm{P}<0.0001)$ and negative expression $(\mathrm{P}<0.0001)$; however, there was no significant difference between low and negative expression $(\mathrm{P}=0.184)$. 
A

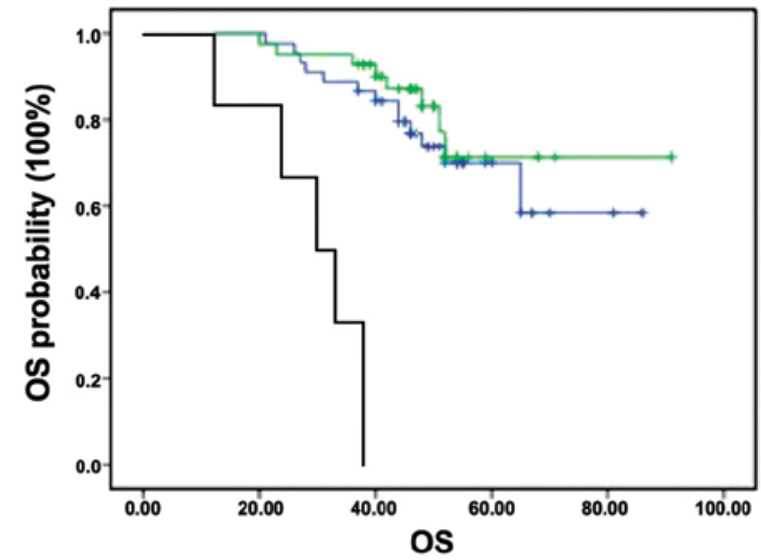

B

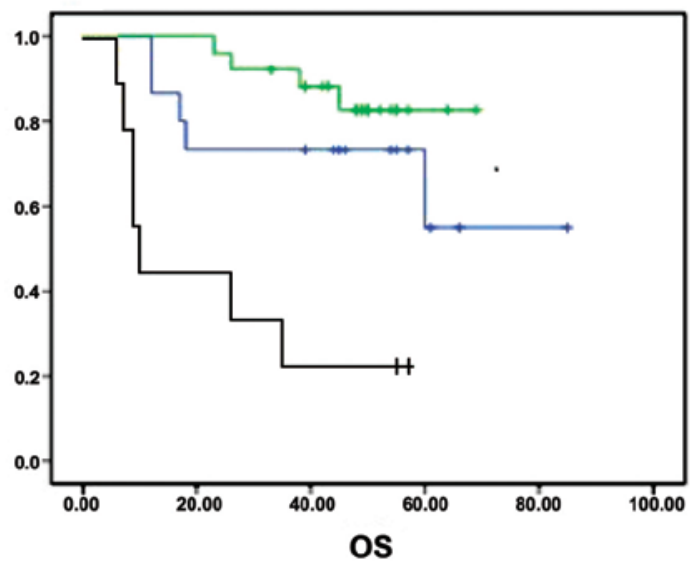

Figure 3. Kaplan-Meier analysis of cancer-specific overall survival (OS) of relapsed patients with stage II colorectal cancer subdivided by the presence of inflammatory adhesions and CXCR3 expression ( $<<0.0001)$. Expression of CXCR3 (A) in cases without adhesions and (B) in cases with adhesions.

be associated with shorter OS and DFS (both P-values $<0.0001$; Fig. 2, Table V). However, CXCL10 expression was significantly associated with DFS $(\mathrm{P}<0.0001)$, but not with OS $(\mathrm{P}=0.181)$ (Table V). Further analysis demonstrated that general classification and the presence of inflammatory adhesions were correlated with CXCR3 $\left(\chi^{2}=7.074, \mathrm{P}=0.029\right)$ and CXCL10 $\left(\chi^{2}=4.863, \mathrm{P}=0.088\right)$. The fact that ulcerated CRCs stained deeper compared with elevated CRCs may be attributed to CXCR3, as one of the common inflammatory cytokines, being involved in ulcer-related inflammation.

Effect of inflammatory adhesions and CXCR3 expression on survival. Of the 401 stage II CRC patients, 91 had inflammatory adhesions, of whom 28 patients developed disease recurrence (Table IA). All the recurrent tumors with inflammatory adhesions were collected for IHC. In view of the results of the log-rank test, we found that the presence of inflammatory adhesions was associated with OS and CXCR3 expression; therefore, the statistical significance of the association was investigated. Under the same conditions of cancer surrounded by adhesions, patients with lower CXCR 3 expression exhibited a better OS. However, patients with the same expression level of CXCR3 and different inflammatory adhesion status exhibited no differences in terms of OS $(\mathrm{P}>0.05)$. On multivariate analysis, only $\mathrm{CXCR} 3$ expression $(\mathrm{P}=0.003)$ was found to be an independent factor predicting a poorer prognosis (Fig. 3).

\section{Discussion}

In this study, we first analyzed the clinicopathological characteristics of stage II CRC and found that colon cancer, a higher number of risk factors, the presence of inflammatory adhesions and tumor size were associated with OS and were independent variables; the presence of inflammatory adhesions, in particular, was found to be a significant factor for recurrent patients. Subsequently, we further investigated the association between the CXCL10/CXCR3 axis and inflammatory adhesions and observed that strong CXCL10 or CXCR3
Table V. Association between CXCL10/CXCR3 and prognosis

\begin{tabular}{lccccc}
\hline & \multicolumn{2}{c}{ DFS $^{\mathrm{a}}$} & & \multicolumn{2}{c}{ OS $^{\mathrm{a}}$} \\
\cline { 2 - 3 } \cline { 5 - 6 } Variables & Mean \pm SE & P-value $^{\mathrm{b}}$ & & Mean \pm SE & P-value $^{\mathrm{b}}$ \\
\hline CXCR3 & & $<0.001^{\mathrm{c}}$ & & $<0.001^{\mathrm{b}}$ \\
expression & & & & \\
Negative & $42.46 \pm 3.30$ & & $67.12 \pm 3.75$ & \\
Low & $44.96 \pm 2.02$ & & $79.52 \pm 3.05$ & \\
High & $11.29 \pm 2.59$ & & $25.21 \pm 4.52$ & \\
CXCL10 & & $<0.001^{\mathrm{c}}$ & & 0.181 \\
expression & & & & \\
Negative & $46.47 \pm 3.41$ & & $68.46 \pm 4.29$ & \\
Low & $51.93 \pm 3.48$ & & $59.83 \pm 2.54$ & \\
High & $28.66 \pm 3.25$ & & $61.79 \pm 5.62$ & \\
\hline
\end{tabular}

${ }^{a}$ DFS and OS calculated as time (months). ${ }^{\text {bP-values were obtained }}$ with the log-rank test. ${ }^{c} \mathrm{P}<0.05$, statistically significant. DFS, disease-free survival; OS, overall survival; SE, standard error.

expression in stage II CRC patients predicted short DFS and OS, particularly CXCR3 expression, which exhibited statistical significance. Furthermore, CXCR3 was found to be closely associated with inflammatory adhesions and OS. We suggest that CXCR3 is a strong indicator of relapse in stage II CRC patients; in addition, CXCR3 was a long-term prognostic biomarker for relapsed stage II patients. The chemokine axis CXCL10/CXCR3 may be the molecular mechanism underlying the development of inflammatory adhesions.

The morbidity and mortality of CRC are on the increase worldwide. Chemokines, as inflammatory cytokines, were first investigated in the context of hematological diseases (13), such as primary thrombocythemia, leukemia, multiple myeloma and von Willebrand syndrome. The association of chemokines with cancer has been attracting increasing atten- 
tion. Schimanski et al (14) and Ottaiano et al (15) used IHC to detect CXCL12/CXCR4 expression in CRC and reported significantly higher expression in stage III/IV compared with stage I/II disease, suggesting that high expression was associated with lymph node and distant metastasis. Similar findings were reported for CCR7 in CRC (16,17), breast cancer (18) and pancreatic cancer (19). Chemokines in tumors mainly function in two ways: They either alter tumor cell actin aggregation, increase or decrease the formation of pseudopodia and affect tumor cell migration, or they promote the secretion of certain cytokines, such as metalloproteinases, degrade the extracellular matrix, damage the endothelial cells and alter vascular permeability, thereby affecting the process of invasion or metastasis.

CXCL10 belongs to the ELR (Glu-Leu-Arg) motif-negative subfamily and acts as an angiogenesis inhibitor; it has also been characterized as a prognostic marker predicting clinical outcome in uterine cervical cancer (20) and melanoma (21). It has been demonstrated that CXCL10 exerts its antitumor effect through its immune-stimulating and angiostatic properties. Of note, the additional roles of CXCL10 in the tumor microenvironment are also important. CXCL10 may activate RhoA and Racl and trigger migration of cancer cells (22). The CXCL10/CXCR3-mediated chemotaxis was found to promote lymph node metastasis in CRC by Kawada et al (9). As one of the first immune defense components, CXCL10 levels increase sharply in the liver, lungs and lymph nodes in CRC by combining to its specific receptor CXCR3. In order to elucidate the mechanism of action, previous researchers constructed a CRC cell metastasis model and found that cells from both primary and metastatic lesions expressed increased levels of CXCL10 and CXCR3 $(23,24)$; they confirmed that tumor cells are able to activate and increase the level of lymphocytes in the microenviroment, upregulate the expression of interferon- $\gamma$ and promote secretion of chemokine axes such as CXCL10/CXCR3, which is a cascade reaction. The underlying molecular mechanism may be CXCL10 promoting the CRC cells to secrete matrix metalloproteinases (MMPs) by inhibiting extracellular signal-regulated kinase 1/2 (ERK1/2) phosphorylation and repressing the ERK signaling pathway. The MMPs may initiate other metastasis-related pathways or alter the adhesive properties of cancer cells, directly promoting invasion. Metzner et al (25) previously analyzed the expression of chemokines in melanoma cells by flow cytometric measurements, ELISA and reverse transcription polymerase chain reaction; they hypothesized that constitutive chemokine expression enables an autocrine growth mechanism in epidermoid carcinoma cells. Numorous studies have demonstrated that CRC cells have the ability to secrete CXCL10 and CXCR3, whereas normal epithelial cells may not express CXCR3. In the present study, although CXCL10 as well as CXCR3 differed between RG and NRG, CXCL10 did not statistically significantly affect DFS or OS. As mentioned above, we hypothesized that the main function of CXCL10 expressed by stage II CRC cells is to promote the secretion of CXCR3 in an autocrine manner and initiate a cascade reaction, promoting invasion and distant migration of malignant carcinoma cells.

Regardless of the local or systemic inflammatory response, cancer-associated inflammation, another studying point, appears to be associated with tumor formation, progression and metastasis and may be of prognostic value in patients with CRC. Hu et al (26) found that microbiota in the gastrointestinal tract may enhance the expression of chemokine CCL5 and induce CCL5-mediated inflammation, which in turn promotes epithelial cell proliferation through local activation of the interleukin 6 (IL-6) pathway, leading to tumorigenesis. Components of the gut microbiota may be associated with individual susceptibility. Through measuring IL-6, IL-10, neutrophil-lymphocyte ratio, neutrophil count and other inflammatory factors, it was previously demonstrated that elevated circulating IL-6 concentration is associated with tumor necrosis (27). The key pathway may be Wnt signaling recognized by the transcription factor nuclear factor- $\mathrm{\kappa B}(28)$. There are no studies on the latent function of peritumoral imflammatory adhesions; however, the association between CXCR3 and inflammation is clear. Dysregulation of CXCR3 expression has been found in viral infections (29), autoimmune diseases (30), allergy and asthma. To the best of our knowledge, the present study is the first to provide supportive evidence for the hypothesis that CRC prognosis is associated with the presence of inflammatory adhesions and CXCR3 expression. In turn, the CXCL10/CXCR3 axis may participate in the peritumoral inflammatory response and promote tumor progression.

In conclusion, we observed that CXCL10 and CXCR3 are upregulated in recurrent CRC tissues. CXCR3 expression may be used as one of the predictors of prognosis in postoperative stage II CRC patients. The cause of adhesion formation remains unclear, but may pertain to the upregulated expression of CXCR3. Therefore, postoperative stage II CRC patients exhibiting strong expression of CXCR3 should be closely followed up.

\section{References}

1. Jemal A, Bray F, Center MM, Ferlay J, Ward E and Forman D: Global cancer statistics. CA Cancer J Clin 61: 69-90, 2011.

2. Chambers AF, Groom AC and MacDonald IC: Dissemination and growth of cancer cells in metastatic sites. Nat Rev Cancer 2: 563-572, 2002

3. Benson AB III, Schrag D, Somerfield MR, Cohen AM, Figueredo AT, Flynn PJ, Krzyzanowska MK, Maroun J, McAllister P, Van Cutsem E, et al: American Society of Clinical Oncology recommendations on adjuvant chemotherapy for stage II colon cancer. J Clin Oncol 22: 3408-3419, 2004.

4. Ribic CM, Sargent DJ, Moore MJ, et al: Tumor microsatellite-instability status as a predictor of benefit from fluorouracil-based adjuvant chemotherapy for colon cancer. N Engl J Med 349: 247-257, 2003.

5. Sargent DJ, Marsoni S, Monges G, Thibodeau SN, Labianca R, Hamilton SR, French AJ, Kabat B, Foster NR, Torri V, et al: Defective mismatch repair as a predictive marker for lack of efficacy of fluorouracil-based adjuvant therapy in colon cancer. J Clin Oncol 28: 3219-3226, 2010.

6. Zlotnik A: Chemokines in neoplastic progression. Semin Cancer Biol 14: 181-185, 2004.

7. Balkwill F: Cancer and the chemokine network. Nat Rev Cancer 4: 540-550, 2004.

8. Dwinell MB,Lügering N,Eckmann Land Kagnoff MF: Regulated production of interferon-inducible T-cell chemoattractants by human intestinal epithelial cells. Gastroenterology 120: 49-59, 2001.

9. Kawada K, Hosogi H, Sonoshita M, Sakashita H, Manabe T, Shimahara Y, Sakai Y, Takabayashi A, Oshima M and Taketo MM: Chemokine receptor CXCR3 promotes colon cancer metastasis to lymph nodes. Oncogene 26: 4679-4688, 2007.

10. Zhang R, Zhang H, Zhu W, Pardee AB, Coffey RJ Jr and Liang P: Mob-1, a Ras target gene, is overexpressed in colorectal cancer. Oncogene 14: 1607-1610, 1997. 
11. Jiang Z, Xu Y and Cai S: CXCL10 expression and prognostic significance in stage II and III colorectal cancer. Mol Biol Rep 37: 3029-3036, 2010.

12. $\mathrm{Li}$ ZS and Li Q: The latest 2010 WHO classification of tumors of digestive system. Zhonghua Bing Li Xue Za Zhi 40: 351-354, 2011 (In Chinese)

13. Matsuda T, Seki T, Ogawara M, Miura R, Yokouchi M and Murakami M: Levels of beta-thromboglobulin and platelet factor 4 in various diseases (author's transl). J Jpn Hematol Soc 43: 871-878, 1980 (In Japanese).

14. Schimanski CC, Schwald S, Simiantonaki N, Jayasinghe C, Gönner U, Wilsberg V, Junginger T, Berger MR, Galle PR and Moehler M: Effect of chemokine receptors CXCR4 and CCR7 on the metastatic behavior of human colorectal cancer. Clin Cancer Res 11: 1743-1750, 2005.

15. Ottaiano A, Franco R, Aiello Talamanca A,LiguoriG, Tatangelo F, Delrio P, Nasti G, Barletta E, Facchini G, Daniele B, et al: Overexpression of both CXC chemokine receptor 4 and vascular endothelial growth factor proteins predicts early distant relapse in stage II-III colorectal cancer patients. Clin Cancer Res 12: 2795-2803, 2006.

16. Correale P, Rotundo MS, Botta C, Del Vecchio MT, Ginanneschi C, Licchetta A, Conca R, Apollinari S, De Luca F, Tassone $\mathrm{P}$, et al: Tumor infiltration by $\mathrm{T}$ lymphocytes expressing chemokine receptor 7 (CCR7) is predictive of favorable outcome in patients with advanced colorectal carcinoma. Clin Cancer Res 18: 850-857, 2012.

17. Li J, Sun R, Tao K and Wang G: The CCL21/CCR7 pathway plays a key role in human colon cancer metastasis through regulation of matrix metalloproteinase-9. Dig Liver Dis 43: 40-47, 2011.

18. Cabioglu N, Yazici MS, Arun B, Broglio KR, Hortobagyi GN, Price JE and Sahin A: CCR7 and CXCR4 as novel biomarkers predicting axillary lymph node metastasis in $\mathrm{T} 1$ breast cancer. Clin Cancer Res 11: 5686-5693, 2005

19. Nakata B, Fukunaga S, Noda E, Amano R, Yamada N and Hirakawa K: Chemokine receptor CCR7 expression correlates with lymph node metastasis in pancreatic cancer. Oncology 74: 69-75, 2008

20. Sato E, Fujimoto J, Toyoki H, Sakaguchi H, Alam SM, Jahan I and Tamaya T: Expression of IP-10 related to angiogenesis in uterine cervical cancers. Br J Cancer 96: 1735-1739, 2007.

21. Antonicelli F, Lorin J, Kurdykowski S, et al: CXCL10 reduces melanoma proliferation and invasiveness in vitro and in vivo. $\mathrm{Br}$ J Dermatol 164: 720-728, 2011.
22. Robledo MM, Bartolome RA, Longo N, Rodríguez-Frade JM, Mellado M, Longo I, van Muijen GN, Sánchez-Mateos P and Teixidó J: Expression of functional chemokine receptors CXCR3 and CXCR4 on human melanoma cells. J Biol Chem 276: 45098-45105, 2001.

23. Zipin-Roitman A, Meshel T, Sagi-Assif O, Shalmon B, Avivi C, Pfeffer RM, Witz IP and Ben-Baruch A: CXCL10 promotes invasion-related properties in human colorectal carcinoma cells. Cancer Res 67: 3396-3405, 2007.

24. Kawada K, Hasegawa S, Murakami T, Itatani Y, Hosogi H, Sonoshita M, Kitamura T, Fujishita T, Iwamoto M, Matsumoto T, et al: Molecular mechanisms of liver metastasis. Int J Clin Oncol 16: 464-472, 2011.

25. Metzner B,Hofmann C,Heinemann C,Zimpfer U,Schraufstätter I, Schöpf E and Norgauer J: Overexpression of CXC-chemokines and CXC-chemokine receptor type II constitute an autocrine growth mechanism in the epidermoid carcinoma cells KB and A431. Oncol Rep 6: 1405-1410, 1999.

26. Hu B, Elinav E, Huber S, Strowig T, Hao L, Hafemann A, Jin C, Wunderlich C, Wunderlich T, Eisenbarth SC, et al: Microbiota-induced activation of epithelial IL-6 signaling links inflammasome-driven inflammation with transmissible cancer. Proc Natl Acad Sci USA 110: 9862-9867, 2013.

27. Guthrie GJ, Roxburgh CS, Richards CH, Horgan PG and McMillan DC: Circulating IL-6 concentrations link tumour necrosis and systemic and local inflammatory responses in patients undergoing resection for colorectal cancer. $\mathrm{Br} \mathrm{J}$ Cancer 109: 131-137, 2013.

28. Vaiopoulos AG, Athanasoula KCh, Papavassiliou AG. NF-кB in colorectal cancer. J Mol Med (Berl): 1029-1037, 2013.

29. de Niet A, de Bruijne J, Plat-Sinnige MJ, Takkenberg RB, van Lier RA, Reesink HW and van Leeuwen EM: Upregulation of CXCR3 expression on $\mathrm{CD} 8^{+} \mathrm{T}$ cells due to the pervasive influence of chronic hepatitis B and $\mathrm{C}$ virus infection. Hum Immunol 74: 899-906, 2013.

30. Björkander S, Heidari-Hamedani G, Bremme K, Gunnarsson I and Holmlund U: Peripheral monocyte expression of the chemokine receptors CCR2, CCR5 and CXCR3 is altered at parturition in healthy women and in women with systemic lupus erythematosus. Scand J Immunol 77: 200-212, 2013. 